\section{Intoxicación por ácido acetilsalicílico, fisiopatología y manejo}

\author{
RODRIGO A. SEPÚLVEDA ${ }^{1}$, MARCOS ORTEGA $^{2}$, \\ NATALIA DONOSO $^{1,3}$, AQUILES JARA ${ }^{1}$
}

\section{Physiopathology and management of acetylsalicylic acid intoxication}

Acetylsalicylic acid (ASA) intoxication is potentially lethal. After ingestion, $A A S$ is rapidly transformed into salicylic acid that dissociates into an hydrogen ion plus salicylate. Salicylate is the main form of AAS in the body and produces multiple alterations. Initially, the stimulation of the ventilatory center promotes a respiratory alkalosis. Then, the mitochondrial dysfunction induced by salicylate, will generate a progressive metabolic acidosis due to the accumulation of ketoacids, lactic acid and dicarboxylic acids among others. Another alterations include hydro electrolytic disorders, gastrointestinal lesions, neurological involvement, ototoxicity and coagulopathy. The correct handling of acetylsalicylic acid intoxication requires an thorough knowledge of its pharmacokinetics and pharmacodynamics. Treatment consists in life support measures, gastric lavage, activated charcoal and urinary alkalization to promote the excretion of salicylates. In some occasions, it will be necessary to start renal replacement therapy as soon as possible.

(Rev Med Chile 2018; 146: 1309-1316)

Key words: Aspirin; Drug Overdose; Salicylates; Therapeutics.
'Departamento de Nefrología, Facultad de Medicina, Escuela de Medicina Pontificia Universidad Católica de Chile. Santiago, Chile. ${ }^{2}$ Enfermedades Respiratorias del Adulto y Departamento de Medicina Intensiva. Pontificia Universidad Católica de Chile. Santiago, Chile.

${ }^{3} U$ nidad de Procedimientos Nefrológicos, Hospital Clínico Universidad Católica-Red de Salud UC Christus. Santiago, Chile.

Los autores declaran que no hubo aportes financieros externos en la realización de esta revisión, ni conflictos de interés.

Recibido el 21 de noviembre de 2017, aceptado el 23 de octubre de 2018.

Correspondencia a: Dr. Rodrigo A. Sepúlveda Departamento de Nefrología. Diagonal Paraguay 362, piso 4. Santiago, Chile. rrsepulveda@uc.cl

\section{L} a intoxicación por salicilatos generalmente ocurre por ingestión de ácido acetilsalicílico (AAS) en forma accidental o como intento suicida. Luego de su ingestión, el AAS se transforma en ácido salicílico. Este ácido se disocia en un hidrogenión y su base conjugada: el anión salicilato. El salicilato es la principal forma que toma el AAS en el organismo y produce severas alteraciones en la función celular, pudiendo generar falla orgánica múltiple y muerte ${ }^{1-5}$. Múltiples factores farmacocinéticos, como dosis, presentación del fármaco ingerido, $\mathrm{pH}$ sistémico, unión a proteínas plasmáticas, biotransformación hepática, función renal y niveles plasmáticos de salicilato; determinarán su volumen de distribución, y por tanto, el ingreso hacia las células. La afectación mitocondrial es lo que produce el daño en la intoxicación por salicilatos. Inicialmente existe una alcalosis respiratoria, pero conforme progresa el cuadro clínico, sobreviene una acidosis metabólica por acumulación de ácido láctico, cetoácidos, ácidos dicarboxílicos, y en menor medida, el ácido salicílico. También existirán alteraciones hidroelectrolíticas, de la coagulación, afectación neurológica que puede ser severa, lesiones gastrointestinales y compromiso cardiopulmonar ${ }^{5}$. La letalidad de una intoxicación severa por salicilatos varía desde $5 \%$ hasta $15 \%{ }^{5}$. En intoxicaciones severas, que requieren manejo de paciente crítico; $61 \%$ de los casos presenta compromiso de conciencia, $47 \%$ falla respiratoria, $36 \%$ acidosis, $14 \%$ inestabilidad hemodinámica y $3 \%$ convulsiones ${ }^{6}$.

El tratamiento consiste en medidas de soporte vital además de favorecer la eliminación del salicilato; esto último se realiza mediante alcalinización urinaria, y en algunos casos, hemodiálisis precoz ${ }^{7,8}$.

El término salicilato, también se utiliza para referirse a la familia de moléculas derivadas del 
ácido salicílico (ácido 2-hidroxibenzoico), aunque en estricto rigor es solo la base conjugada de éste. El salicilato, también puede provenir de la metabolización de otras moléculas como el ácido salicílico (extracto de corteza de Sauce y Filipendula entre otras hierbas), metilsalicilato (aceite de gaulteria), ácido 5-aminosalicílico (mesalazina), salicilamida y sales de salicilato. Debido al enorme uso y disponibilidad del AAS es la principal molécula que genera intoxicación por salicilato. La mesalazina, se metaboliza mínimamente a salicilato y las otras moléculas son poco asequibles. En esta revisión discutiremos el enfrentamiento y manejo de la intoxicación por salicilato debida a la ingestión excesiva de AAS. Para este objetivo es necesario comprender la farmacocinética y farmacodinamia del AAS y el salicilato, conocer el cuadro clínico y repercusiones sistémicas asociadas al salicilato, además de saber cómo instaurar un tratamiento adecuado y oportuno.

\section{Farmacocinética}

El AAS en bajas dosis $(<100 \mathrm{mg} / \mathrm{d})$ bloquea irreversiblemente la enzima ciclooxigenasa-1 (COX-1) evitando la formación de tromboxano. Esto inhibe la agregación plaquetaria y produce un efecto antitrombótico. Mayores dosis $(<4 \mathrm{~g} / \mathrm{d})$ bloquean ambas enzimas, COX-1 y COX-2, generando un efecto analgésico y antipirético. Dosis muy altas (4-8 g/d) son anti-inflamatorias, estas dosis pueden llegar a producir niveles plasmáticos de salicilato $>300 \mu \mathrm{g} / \mathrm{mL}$.

El nivel plasmático de salicilato que se correlaciona con actividad terapéutica del AAS oscila entre $50-100 \mu \mathrm{g} / \mathrm{mL}$ para un efecto analgésico y entre $100-200 \mu \mathrm{g} / \mathrm{mL}$ para un efecto anti-inflamatorio ${ }^{5}$. Niveles $>300 \mu \mathrm{g} / \mathrm{mL}$ se asocian con aparición de tinitus, vértigo y polipnea. Niveles más elevados producen alteración del estado mental, hipertermia, déficit neurológico, convulsiones, coma, edema pulmonar, hipotensión, falla renal aguda y trastornos hidroelectrolíticos severos ${ }^{1-3}$ (Tabla 1).

Una ingestión aguda de AAS $<150 \mathrm{mg} / \mathrm{kg}$ no produce reacciones tóxicas; $150-300 \mathrm{mg} / \mathrm{kg}$ pueden generar una intoxicación leve a moderada, pero el consumo de $300-500 \mathrm{mg} / \mathrm{kg}$ produce una intoxicación severa ${ }^{9}$. La ingestión $>500 \mathrm{mg} / \mathrm{kg}$ es potencialmente letal.

El AAS se transforma en ácido salicílico (AS) mediante hidrólisis por esterasas. AAS y AS son ácidos fuertes ( $\mathrm{pKa}=3,49$ y $\mathrm{pKa}=2,97$ respectivamente), por lo tanto, en el agua corporal entregarán $\mathrm{H}^{+}$y su base conjugada (acetilsalicilato y salicilato respectivamente). Los compuestos aniónicos (acetilsalicilato y salicilato) son más hidrosolubles, mientras los ácidos (ASA y AS), que no poseen carga, son más liposolubles.

Después de la ingestión de AAS, una porción es hidrolizada por esterasas en la mucosa gástrica e intestinal. Las formas no-ionizadas, y por tanto, más liposolubles (ASA y AS), son absorbidas desde el estómago. Esto se favorece por un $\mathrm{pH}$ ácido que tiende a desplazar la reacción de los ácidos hacia los reactantes. El intestino delgado, gracias a su enorme superficie, es el sitio de mayor absorción, sobre todo cuando los comprimidos tienen cubierta entérica ya que se degradan con $\mathrm{pH}$ alcalino ${ }^{3,4}$.

La velocidad de absorción del AAS y sus derivados está determinada por factores como: dosis, disolución de tabletas, $\mathrm{pH}$ en mucosa entérica y tiempo de vaciado gástrico ${ }^{3}$. La acumulación de tabletas con cobertura entérica genera bezoares estomacales que retardan el tiempo de absorción o pueden romperse provocando una intoxicación masiva $^{1,10}$. A dosis terapéuticas, AAS y AS son absorbidos en 15-30 min luego de su ingesta,

Tabla 1. Síntomas según severidad de intoxicación ${ }^{5}$

\begin{tabular}{|lcl|}
\hline Severidad & Salicilato en plasma $(\boldsymbol{\mu g} \mathbf{m} \mathbf{m L})$ & Síntomas \\
Leve & $300-600$ (adulto) & Náuseas, vómitos, dolor abdominal, tinitus, polipnea \\
& $200-450$ (infante) & Mareo, letargia, polipnea \\
Moderado & $600-800$ (adulto) & $\begin{array}{l}\text { Agrega sudoración hipertermia } \\
\text { Agrega deshidratación, descoordinación, intranquilidad }\end{array}$ \\
Severo & $450-700$ (infante) & Agrega hipotensión \\
& $>800$ (adulto) & $\begin{array}{l}\text { Agrega sangrados, lesiones purpúricas, alucinaciones, estupor, coma, } \\
\text { falla renal, edema pulmonar }\end{array}$ \\
\hline
\end{tabular}


y la concentración máxima se detecta luego de 1-2 $h^{3}$. Una sobredosis prolonga el vaciamiento gástrico, retardando la concentración máxima a 18-24 h, incluso a $60 \mathrm{~h}$ cuando existe un bezoar estomacal ${ }^{1,3,10}$.

Hasta el 80\% de una dosis de AAS ingerida será absorbida, pero el primer paso hepático producirá que solo $45-50 \%$ de la dosis inicial alcance la circulación sistémica ${ }^{5}$. Finalmente, esterasas en el plasma, hígado, plaquetas y eritrocitos; hidrolizan el AAS y solo una mínima porción mantendrá la forma acetilada ${ }^{3}$. Esta biotransformación no es saturable y ocurre en pocos minutos ${ }^{5}$. Consecuentemente la principal forma del AAS será el salicilato (Figura 1).

El volumen de distribución (Vd) del salicilato dependerá de su concentración plasmática, niveles de albúmina, $\mathrm{pH}$ sistémico y competencia con otras drogas por unión a proteínas. En sujetos sanos, con dosis terapéuticas, el Vd es bajo, $\approx 0,2$ $\mathrm{L} / \mathrm{kg}^{3,5}$. Con dosis mayores, se saturan los sitios de unión a proteínas, aumentando el $\mathrm{Vd} \mathrm{a} \approx 0,5 \mathrm{~L} / \mathrm{kg}^{5}$. A bajas concentraciones de salicilato $(<100 \mu \mathrm{g} /$ $\mathrm{mL}), 90 \%$ está unido a albúmina, pero en concentraciones altas $(>400 \mu \mathrm{g} / \mathrm{mL})$, solo $75 \%$. Pacientes hipoalbuminémicos tienen mayor Vd del salicilato. La acidemia favorece la forma liposoluble incrementando el Vd. En alcalosis predomina la forma hidrosoluble (salicilato) disminuyendo el $\mathrm{Vd}$. A $\mathrm{pH}=7$ la relación salicilato/AS es $\approx 10.000$; y a $\mathrm{pH} 7,4$ es $\approx 50.000$. Por esta razón, un $\mathrm{pH}$ sanguíneo básico evita que el salicilato atraviese membranas plasmáticas (como la barrera hematoencefálica); y la alcalinización urinaria favorece su excreción renal (evitando la reabsorción del componente liposoluble).

La biotransformación del AAS permitirá que se convierta a metabolitos inactivos y más hidrosolubles favoreciendo su eliminación. Inicialmente ocurre mediante hidrólisis por esterasas, y posteriormente, tendrá lugar la conjugación hepática del salicilato.

En el hígado, mediante conjugación con glicina, el salicilato se transforma en salicilurato. Existe, también, conjugación hepática con ácido glucurónico, pero en una mínima proporción. En dosis terapéuticas, la biotransformación hepática del salicilato ocurre siguiendo una cinética de primer orden ${ }^{3}$. La reacción con glicina se satura a concentraciones de salicilato $>300 \mu \mathrm{g} / \mathrm{mL}^{5}$. Esto significa que una sobredosis convierte la metabolización hepática del salicilato en un proceso cinético de orden cero, favoreciendo su toxicidad ${ }^{3,11}$.

La excreción del salicilato se realiza por vía renal: $75 \%$ se elimina como salicilurato, $10 \%$ como salicilato, $10 \%$ como fenil-salicilato-glucurónico

\section{Ácido Acetilsalicílico $\leftrightarrow$ Acetilsalicilato $+\mathrm{H}^{+}$ \\ Biodisponibilidad 45-50\% de la dosis inicial luego de absorción intestinal y primer paso hepático}

Ácido Acetilsalicílico $\leftrightarrow$ Ácido Salicílico + grupo acetilo
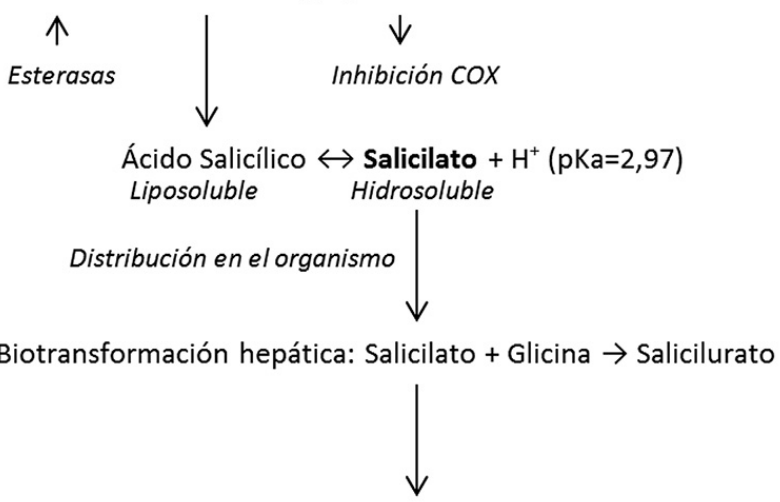

Excreción Renal: $75 \%$ Salicilurato, 10\% Salicilato
Figura 1. Ácido acetilsalicílico en el organismo. 
y 5\% como otros derivados glucurónicos y del salicilurato $^{3,5}$. La excreción renal de salicilato no modificado varía entre $2-30 \%$ según el $\mathrm{pH}$ urinario. Un aumento del pH urinario desde 6 a 8, incrementa 4 veces su excreción ${ }^{12}$.

Por lo tanto, son múltiples los factores que influirán en la vida media del salicilato. La dosis, pH sistémico y proteínas plasmáticas determinarán su Vd. A mayor Vd, mayor vida media. Por otro lado, la biotransformación hepática se satura en sobredosis, nuevamente aumentando la vida media. Con dosis terapéuticas, la vida media del salicilato es 2-3 h, mientras que durante una sobredosis, ésta aumenta a 20-30 h ${ }^{5}$ (Tabla 2).

\section{Farmacodinamia}

Los efectos sistémicos del AAS son mediados por el grupo acetilo y el salicilato. La acetilación ocurre con bajas dosis de AAS y afecta a ambas ciclooxigenasas (COX-1 y COX-2). La COX acetilada no produce prostaglandinas ni tromboxanos, pero la COX-2 acetilada puede generar nuevos productos que presentan efecto anti-inflamatorio y protector endotelial ${ }^{5}$. Los anti-inflamatorios no esteroidales no poseen este efecto benéfico sobre COX-2, y además, compiten con el AAS. La inhibición de la producción de prostaglandinas $y$ tromboxanos es responsable de los efectos anti-plaquetarios, anti-inflamatorios, anti-piréticos, analgésicos y antineoplásicos del AAS.

Otros efectos celulares requieren altas dosis y son mediados por el salicilato. Éste disminuye la transcripción génica de COX-2 e iNOS ${ }^{5,13}$. El salicilato es un constituyente natural de plantas que las protege de noxas exógenas como bacterias o virus ${ }^{5}$, también modula procesos asociados a la inflamación, respuesta inmune y defensa anti-tumoral. Sin embargo, el salicilato puede afectar gravemente el metabolismo energético celular.

\section{Efectos sistémicos de la sobredosis}

En la mitocondria, las moléculas de alto poder reductor $\left(\mathrm{NADH}, \mathrm{FADH}_{2}\right)$ generadas por la glucólisis anaeróbica y ciclo de Krebs, ceden electrones que serán transportados por la cadena de electrones ubicada en la membrana mitocondrial interna. Esto, brinda energía para la acumulación de $\mathrm{H}^{+}$en el espacio intermembrana mitocondrial.

\section{Tabla 2. Elementos farmacocinéticos que favorecen toxicidad por salicilato}

La sobredosis de AAS retarda el vaciamiento gástrico favoreciendo una intoxicación más prolongada y severa

Altas concentraciones de salicilato saturan su unión a proteínas plasmáticas, aumentando el salicilato libre

La acidemia favorece la forma liposoluble (ácido salicílico) aumentando su volumen de distribución y vida media. Al contrario, la alcalosis, favorece la forma hidrosoluble (salicilato) disminuyendo el volumen de distribución, vida media y aumentando la excreción renal

Con niveles de salicilato $>300 \mu \mathrm{g} / \mathrm{mL}$ comienzan los síntomas de intoxicación y se satura la conjugación hepática de éste, convirtiendo la cinética de eliminación en orden 0

La enzima ATP-sintasa utiliza este gradiente de $\mathrm{H}^{+}$para obtener la energía necesaria en la síntesis del ATP. El $\mathrm{O}_{2}$ será quien acepte el $\mathrm{H}^{+}$y electrón, formando agua. El salicilato no bloquea la cadena de electrones (como sí lo hacen otros tóxicos mitocondriales), sino que desacopla la membrana mitocondrial interna disipando el gradiente de concentración de $\mathrm{H}^{+}$. Así, la mitocondria continuará llevando a cabo la reacción, generando calor (hipertermia), agua, $\mathrm{CO}_{2}$; pero no ATP (Figura 2).

La depleción de ATP obligará a la oxidación de glucosa y ácidos grasos en un intento de obtener energía. Esto provocará sobreproducción de $\mathrm{CO}_{2}$. Tanto el exceso de $\mathrm{CO}_{2}$ como el salicilato promoverán hiperventilación en el tronco encefálico. La polipnea comienza con niveles de salicilato en sangre $>300 \mu \mathrm{g} / \mathrm{mL}$ y es significativa a $500 \mu \mathrm{g} / \mathrm{mL}^{5}$. Así, el trastorno ácido-base inicial en intoxicación por AAS es la alcalosis respiratoria.

La depleción de ATP obliga a movilizar glucosa, ácidos grasos y amonioácidos para su combustión. Todos ellos aumentarán en la sangre, por este motivo, inicialmente existirá hiperglicemia. La liberación masiva de catecolaminas (asociada a la necesidad de movilizar nutrientes) puede generar lesión endotelial pulmonar y aumento de la permeabilidad vascular ${ }^{14}$.

Los ácidos grasos de cadena media y corta atraviesan libremente la membrana mitocondrial para su oxidación. Sin embargo, ácidos grasos de cadena larga no pueden debido a las alteraciones producidas por el salicilato. Luego, estos ácidos grasos de cadena larga, se acumularán en la forma de ácidos dicarboxílicos ${ }^{5}$. 


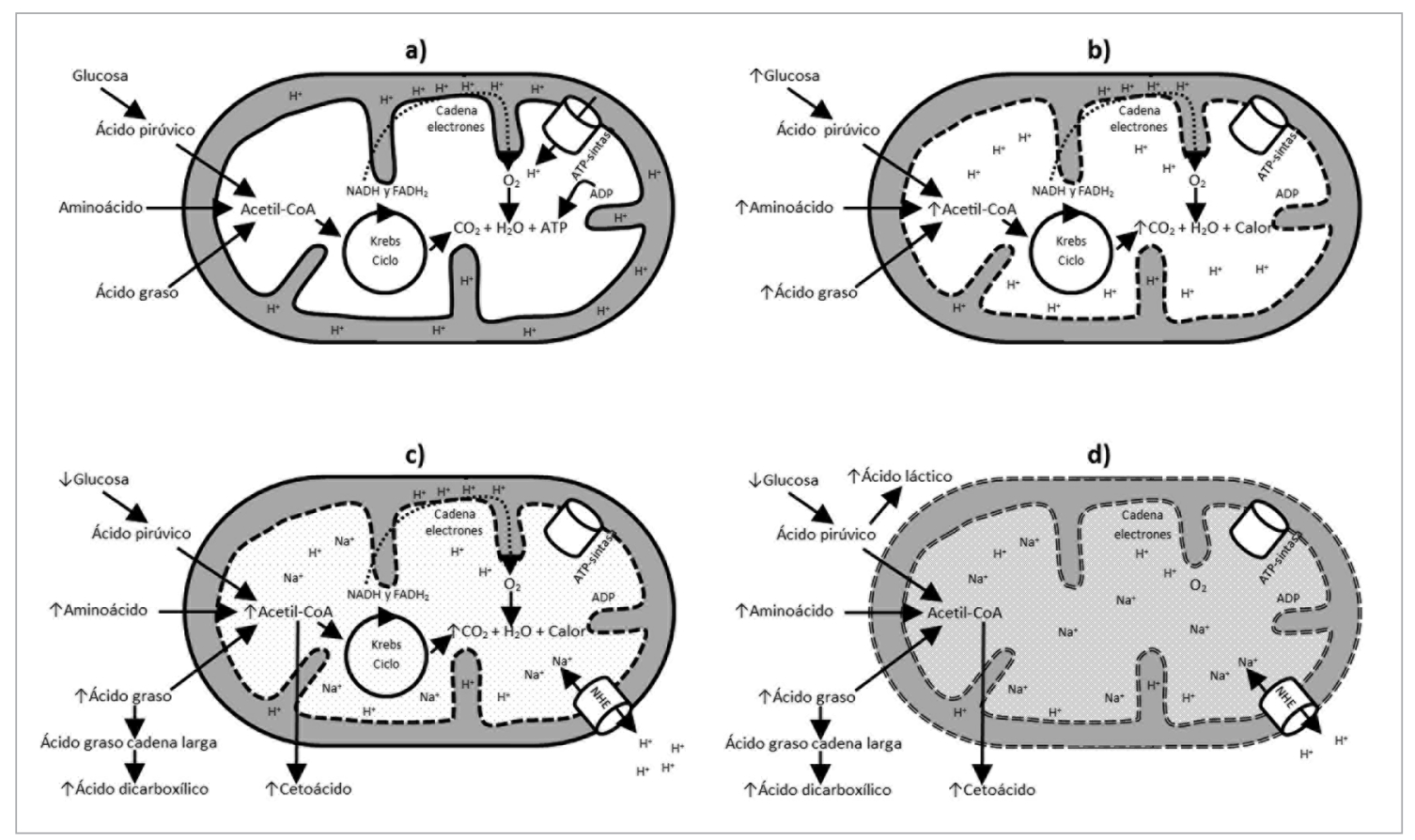

Figura 2. Progresión del daño mitocondrial y alteraciones metabólicas. a) Mitocondria normal: recibe nutrientes para producción de energía. El ciclo de Krebs genera $\mathrm{CO}_{2}$ y moléculas de alto poder reductor $\left(\mathrm{NADH}, \mathrm{FADH} \mathrm{H}_{2}\right)$. Éstas, entregarán su electrón a la cadena de electrones en la membrana mitocondrial interna generando un gradiente de $\mathrm{H}^{+}$en el espacio intermembrana. Este gradiente químico es usado como energía por la ATP-sintasa para producir ATP desde ADP. El aceptor final del $\mathrm{H}^{+}$y electrón es el $\mathrm{O}_{2}$, produciendo $\mathrm{H}_{2} \mathrm{O}$. b) Desacoplamiento de la membrana interna por salicilato: se disipa el gradiente de $\mathrm{H}^{+}$evitando la formación de ATP y la energía se escapa como calor. Comienza la movilización de nutrientes para obtener energía y producción de $\mathrm{CO}_{2}$. c) Producción mitocondrial de ácidos: el exceso de acetil-CoA promueve desarrollo de cetosis. Por la imposibilidad de oxidar ácidos grasos de cadena larga, se acumulan ácidos dicarboxílicos. El transportador $\mathrm{NHE}$ ayuda a remover los $\mathrm{H}^{+}$en exceso incorporando $\mathrm{Na}^{+}$. d) Edema y disfunción mitocondrial: el exceso de $\mathrm{Na}^{+}$genera edema, y así, mayor disfunción mitocondrial. Sobreviene el metabolismo anaeróbico con acidosis láctica.

El exceso de $\mathrm{H}^{+}$en la mitocondria es eliminado mediante antiporte con sodio al citosol. El sodio intramitocondrial genera edema y mayor disfunción. El hepatocito es la célula más afectada en una intoxicación por salicilato. La inhibición del ciclo de la urea produce acumulación de aminoácidos.

En estas condiciones muchas de las funciones mitocondriales están alteradas mientras siguen llegando nutrientes para su oxidación. Este exceso de oxidación de nutrientes favorecerá la generación de acetil-CoA. La sobreutilización de glucosa puede generar hipoglicemia y disminución de insulina. Esta última hormona es necesaria para estimular la enzima piruvato deshidrogenasa. De la misma forma, un exceso de acetil-CoA inhibe la acción de esta enzima. Así, habrá tendencia a la cetosis (por acumulación de acetil-CoA) y acidosis láctica (por predominio de glucólisis anaeróbica para obtener energía).

Con la progresión de la intoxicación por salicilatos, sobreviene una acidosis metabólica con anion gap (AG) aumentado debido a la acumulación de ácido láctico, cetoácidos, ácidos dicarboxílicos y aminoácidos. La participación del ácido salicílico es mínima, de hecho, $1.000 \mu \mathrm{g} / \mathrm{mL}$ de salicilato solo aportan $7 \mathrm{mEq} / \mathrm{L}$ de carga. El salicilato puede interferir en la medición del cloruro produciendo una falsa normalización del $\mathrm{AG}^{15}$.

En población infantil la disfunción mitocondrial y acidosis metabólica ocurren en forma pre$\mathrm{COZ}$, no presentando una fase inicial con alcalosis respiratoria.

Se ha descrito bicarbonaturia en la intoxicación 
por salicilatos ${ }^{16}$, esto podría estar asociado a disfunción tubular proximal por depleción de ATP.

La deshidratación es común y secundaria a vómitos, anorexia, hiperventilación e hiperpirexia; ésta puede llegar a $4-6 \mathrm{~L} / \mathrm{m}^{2}$ en una intoxicación severa $^{10}$. El estado de hipovolemia, alteraciones cardiopulmonares y la disfunción celular contribuyen a la acidosis láctica. La hipernatremia se asocia a pérdidas de agua libre y administración de $\mathrm{NaHCO}_{3}$.

Pese a existir una acidosis, es frecuente la hipokalemia secundaria a pérdidas renales. Esta última se debe a la administración de $\mathrm{NaHCO}_{3}$ intravenoso (bicarbonaturia), eliminación urinaria de aniones (bases conjugadas de los ácidos) e hiperaldosteronismo secundario que "arrastran" potasio en el nefrón. A esto se agrega el estado adrenérgico que favorece su redistribución al intracelular.

El efecto del salicilato en la excreción urinaria de ácido úrico es dual; a bajas concentraciones (50-100 $\mu \mathrm{g} / \mathrm{mL}$ ) actúa como anti-uricosúrico y a altas concentraciones $(>150 \mu \mathrm{g} / \mathrm{mL})$ funciona como uricosúrico. El efecto anti-uricosúrico podría estar mediado por el transportador de aniones orgánicos en la membrana basolateral del túbulo proximal. Mientras, que las altas concentraciones de salicilato inhiben los transportadores URAT1 y URATv1 en la membrana apical del túbulo proximal evitando la reabsorción de ácido úrico ${ }^{17,18}$.

En intoxicaciones severas, el salicilato puede prolongar el tiempo de protrombina afectando la coagulación ${ }^{19,20}$.

Lesiones en la mucosa gástrica van desde erosión superficial, úlceras y sangrados. Las alteraciones en la barrera mucosa permiten retro-difusión de $\mathrm{H}^{+}$con activación de pepsina e injuria celular ${ }^{5}$.

Síntomas típicos y precoces de una intoxicación aguda por salicilatos son tinitus y sordera (principalmente tonos bajos) ${ }^{1,3}$. La afectación es bilateral, simétrica y reversible en 1-3 días luego del retiro de la droga. El salicilato podría afectar canales de cloro en los cilios de las células cocleares $^{1}$. Otros síntomas por ototoxicidad son vértigo, mareo y alteraciones de la marcha ${ }^{5}$.

El mecanismo por el cual el salicilato produce encefalopatía es desconocido. Se ha asociado con disfunción mitocondrial, depleción de ATP, hipoglicemia, hipotensión, acidosis y alteraciones hidroelectrolíticas. El espectro varía desde la confusión al coma. Si la acidosis es severa, aumenta el
AS y cruza la barrera hematoencefálica. El salicilato en el cerebro produciría liberación de factores apoptóticos ${ }^{21}$.

\section{Tratamiento}

El manejo inicial incluye medidas de soporte vital, lavado gástrico, carbón activado y alcalinización urinaria para promover la excreción renal del salicilato $^{3,5,22}$. También es fundamental hidratar y corregir alteraciones hidroelectrolíticas. La hipoglicemia y cetosis podrían requerir administración de glucosa o insulina.

El vómito espontáneo que presentan los pacientes permite evitar absorción de la droga, pero éste no debe ser inducido cuando hay compromiso de conciencia. La utilidad del lavado gástrico es controversial. Se estima que a la hora de ingestión la cantidad de AAS que logra extraer es mínima, por este motivo es discutible utilizarlo después de este período ${ }^{23}$. Se recomienda administrar carbón activado (25-50 g en adultos o $1 \mathrm{~g} / \mathrm{kg}$ en infantes) idealmente antes de que se logre el máximo nivel sérico de salicilato ${ }^{1,5}$. Dosis múltiples (cada 1-4 h) de carbón activado son controversiales y no se han asociado a mayores beneficios ${ }^{24}$. Como el AAS es absorbido rápidamente y su concentración plasmática máxima se logra precozmente, difícilmente será posible administrar más de 1 dosis. El carbón activado no está exento de complicaciones, en dosis altas se ha asociado con íleo, constipación, obstrucción intestinal, vómitos y aspiración ${ }^{24}$.

En la intoxicación por AAS las medidas para prevenir su absorción son poco efectivas, por lo tanto, serán fundamentales terapias que favorezcan la eliminación del salicilato. La depuración renal del salicilato aumenta más con un $\mathrm{pH}$ urinario alto, que con un flujo urinario elevado. La alcalinización urinaria debe ser iniciada con niveles de salicilato $>300 \mu \mathrm{g} / \mathrm{mL}$. Administrar bicarbonato de sodio intravenoso para un $\mathrm{pH}$ urinario $>7,5$; pero sin producir alcalemia $>7,55$. La utilización de acetazolamida es controversial; intensifica la bicarbonaturia aumentando la eliminación urinaria de salicilatos, siendo útil cuando no es posible administrar más $\mathrm{NaHCO}_{3}$ por alcalemia ${ }^{25}$. Sin embargo, otros autores detallan efectos perjudiciales por interacción con salicilato ${ }^{26} \mathrm{o}$ empeoramiento de la acidosis ${ }^{27}$.

El salicilato tiene un bajo $\mathrm{Vd}$, es una molécula 
Intoxicación por ácido acetilsalicílico - R. A. Sepúlveda et al

Tabla 3. Medidas terapéuticas

Iniciar lavado gástrico hasta $1 \mathrm{~h}$ después de la ingestión de AAS

Administrar una dosis de carbón activado (25-50 g en adultos o $1 \mathrm{~g} / \mathrm{kg}$ en infantes) en las primeras horas luego de la ingestión de AAS

Con dosis de AAS $>4 \mathrm{~g} / \mathrm{d}$ o niveles de salicilato $>300 \mu \mathrm{g} / \mathrm{mL}$ se debe iniciar alcalinización urinaria. Administrar NaHCO intra-venoso para un $\mathrm{pH}$ urinario $>7,5$; evitando un $\mathrm{pH}$ plasmático $>7,55$

Indicaciones de hemodiálisis:

- Síntomas de intoxicación severa: compromiso de conciencia, alucinaciones, agitación, coma, convulsiones y edema pulmonar

- Laboratorio de intoxicación severa:

- Adultos: salicilato en sangre $>800 \mu \mathrm{g} / \mathrm{mL}$ o $>600 \mu \mathrm{g} / \mathrm{mL}$ con síntomas significativos

- Infantes y ancianos: salicilato en sangre $>700 \mu \mathrm{g} / \mathrm{mL}$

- Inestabilidad hemodinámica

Falla renal

Acidosis metabólica severa $(\mathrm{pH}<7,2)$ luego de hidratación

Sobrecarga de volumen refractaria a manejo médico

pequeña (peso molecular $138 \mathrm{~g} / \mathrm{mol}$ ), y además, la unión a proteínas plasmáticas disminuye si los niveles son elevados. Estas propiedades favorecen su eliminación mediante hemodiálisis.

Las indicaciones para comenzar hemodiálisis son variables según diversos autores, pero la mayoría coincide en que debe ser iniciada cuando las concentraciones de salicilato son potencialmente letales. Se acepta que pacientes con compromiso hemodinámico, falla renal aguda, alteración neurológica severa y/o acidosis metabólica severa que no responden a terapia médica; deben comenzar terapia de reemplazo renal ${ }^{28,29}$ (Tabla 3 ).

No existe unanimidad respecto a la técnica de depuración extracorpórea que se debe practicar ${ }^{30}$. Warthall et $\mathrm{al}^{31}$ describieron una reducción en los niveles de salicilato entre $77-84 \%$ con hemodiafiltración venovenosa continua, mientras Lund et $\mathrm{al}^{8}$ demostraron resultados similares utilizando hemodiálisis convencional seguida de hemodiálisis continua. La hemoperfusión es efectiva en remover salicilato, pero no corrige las alteraciones ácido-base ni hidroelectrolíticas. La diálisis peritoneal no es un método eficiente para remover salicilato. El inicio precoz de hemodiálisis permite lograr una reducción significativa del tóxico en corto tiempo evitando las graves repercusiones en la función celular. Como en fase inicial, los niveles de salicilato son más elevados (menor unión a proteínas plasmáticas) y la acidosis no se ha establecido totalmente (menor $\mathrm{Vd}$ ); se favorece una mayor extracción del tóxico.

Creemos que es necesario comenzar tratamiento mediante hemodiálisis en aquellos casos de gravedad o importante repercusión clínica o de laboratorio, para lograr una reducción significativa del tóxico en un corto tiempo. Posteriormente, de acuerdo a la evolución clínica y las concentraciones séricas de salicilatos, se podrán realizar otras técnicas depurativas, según las necesidades del paciente.

\section{Referencias}

1. O’Malley GF. Emergency department management of the salicylate-poisoned patient. Emerg Med Clin North Am 2007; 25 (2): 333-46.

2. Minns AB, Cantrell FL, Clark RF. Death due to acute salicylate intoxication despite dialysis. J Emerg Med 2011; 40 (5): 515-7.

3. Grosser T, Smyth E, Fitz Gerald GA. Anti-Inflammatory, Antipyretic, and Analgesic Agents; Pharmacotherapy of Gout. In: Goodman \& Gilman's: The Pharmacological Basis of Therapeutics, 12th ed. New York, McGraw-Hill Medical; 2011: 959-1004.

4. American College of Medical Toxicology. Guidance Document: Management Priorities in Salicylate Toxicity. J Med Toxicol 2015; 11 (1): 149-52.

5. Schrör K. Acetylsalicylic Acid. Primera edición. Weinheim: Wiley-Blackwell; 2009. 
6. Thisted B, Krantz T, Strøom J, Sørensen MB. Acute salicylate self-poisoning in 177 consecutive patients treated in ICU. Acta Anaesthesiol Scand 1987; 31 (4): 312-6.

7. Locket $S$. Haemodialysis in the treatment of acute poisoning. Proc R Soc Med 1970; 63 (5): 427-30.

8. Lund B, Seifert SA, Mayersohn M. Efficacy of sustained low-efficiency dialysis in the treatment of salicylate toxicity. Nephrol Dial Transplant 2005; 20 (7): 1483-4.

9. Temple AR. Acute and chronic effects of aspirin toxicity and their treatment. Arch Intern Med 1981; 141 (3): 364-9.

10. Poisoning with enteric-coated aspirin. Lancet 1981; 2 (8238): 130.

11. Levy G. Pharmacokinetics of salicylate elimination in man. J Pharm Sci 1965; 54 (7): 959-67.

12. Prescott LF, Balali-Mood M, Critchley JA, Johnstone AF, Proudfoot AT. Diuresis or urinary alkalinisation for salicylate poisoning? Br Med J 1982; 285 (6352): 1383-6.

13. Xu X-M, Sansores-García L, Chen X-M, Matijevic-Aleksic N, Du M, Wu KK. Suppression of inducible cyclooxygenase 2 gene transcription by aspirin and sodium salicylate. Proc Natl Acad Sci USA 1999; 96 (9): 5292-7.

14. Yuklyaeva N, Chaudhary A, Gorantla R, Bischof E. Salicylate-induced pulmonary edema-a near-miss diagnosis. Am J Emerg Med 2014; 32 (5): 490-6.

15. Jacob J, Lavonas EJ. Falsely normal anion gap in severe salicylate poisoning caused by laboratory interference. Ann Emerg Med 2011; 58 (3): 280-1.

16. Tsimihodimos V, Psychogios N, Kakaidi V, Bairaktari E, Elisaf M. Salicylate-induced proximal tubular dysfunction. Am J Kidney Dis 2007; 50 (3): 463-7.

17. Nakanishi T, Ohya K, Shimada S, Anzai N, Tamai I. Functional Cooperation of URAT1 (SLC22A12) and URATv1 (SLC2A9) in Renal Reabsorption of Urate. Nephrol Dial Transplant 2013; 28: 603-11.

18. Ohtsu N, Anzai N, Fukutomi T, Kimura T, Sakurai H, Endou H. Human renal urate transpoter URAT1 mediates the transport of salicylate. Nihon Jinzo Gakkai Shi 2010; 52 (4): 499-504.

19. Ayalon I, Alder MN, Langner TR, Hafberg ET, Miethke AG, Kaplan JM. A Case of Salicylate Intoxication Complicated by Coagulopathy, Pulmonary Edema, and Pancreatitis. Am J Ther 2016; 23 (6): 1929-32.

20. Park BK, Leck JB. On the mechanism of salicylate-indu- ced hypothrombinaemia. J Pharm Pharmacol 1981; 33 (1): 25-8.

21. Rauschka H, Aboul-Enein F, Bauer J, Nobis H, Lassmann H, Schmidbauer M. Acute cerebral white matter damage in lethal salicylate intoxication. Neurotoxicology 2007; 28 (1): 33-7.

22. Chyka PA, Erdman AR, Christianson G, Wax PM, Booze LL, Manoguerra AS, et al. Salicylate poisoning: an evidence-based consensus guideline for out-of-hospital management. Clin Toxicol (Phila) 2007; 45 (2): 95-131.

23. Vale JA, Kulig K. Position paper: gastric lavage. J Toxicol Clin Toxicol 2004; 42 (7): 933-43.

24. American Academy of Clinical Toxicology; European Association of Poisons Centres and Clinical Toxicologists. Position statement and practice guidelines on the use of multi-dose activated charcoal in the treatment of acute poisoning. J Toxicol Clin Toxicol 1999; 37 (6): 731-51.

25. DuBose TD. Disorders of Acid-Base Balance. En: Skorecki K, Chertow GM, Marsden PA, Taal MW, Yu A, editores, Brenner and Rector's the kidney. Philadelphia: Elsevier; 2016. p. 547-48.

26. Sweeney KR, Chapron DJ, Brandt JL, Gomolin IH, Feig PU, Kramer PA. Toxic interaction between acetazolamide and salicylate: case reports and a pharmacokinetic explanation. Clin Pharmacol Ther 1986; 40 (5): 518-24.

27. Cartwright MM, Hajja W, Al-Khatib S, Hazeghazam M, Sreedhar D, Li RN, et al. Toxigenic and metabolic causes of ketosis and ketoacidotic syndromes. Crit Care Clin 2012; 28 (4): 601-31.

28. Mokhlesi B, Leikin JB, Murray P, Corbridge TC. Adult toxicology in critical care: Part II: specific poisonings. Chest 2003; 123 (3): 897-922.

29. Dargan P, Wallace C, Jones A. An evidence based flowchart to guide the management of acute salicylate (aspirin) overdose. Emerg Med J 2002; 19 (3): 206-9.

30. Juurlink DN, Gosselin S, Kielstein JT, Ghannoum M, Lavergne V, Nolin TD, et al. Extracorporeal Treatment for Salicylate Poisoning: Systematic Review and Recommendations From the EXTRIP Workgroup. Ann Emerg Med 2015; 66 (2): 165-81.

31. Wrathall G, Sinclair R, Moore A, Pogson D. Three case reports of the use of haemodiafiltration in the treatment of salicylate overdose. Hum Exp Toxicol 2001; 20 (9): 491-5. 